\title{
Investigation of Synchronizations in Five Cross-Coupled Chaotic Circuits
}

\author{
Naoto Kageyama, Yoko Uwate and Yoshifumi Nishio \\ $†$ Dept. of Electrical and Electronic Eng., Tokushima University \\ 2-1 Minami-Josanjima, Tokushima, 770-8506 JAPAN \\ Email : \{n-kage, uwate, nishio\}@ee.tokushima-u.ac.jp
}

\begin{abstract}
Studies on chaos synchronization in coupled chaotic circuits are extensively carried out in various fields. In this study, we investigate synchronization phenomena observed in five simple chaotic circuits cross-coupled by five inductors. Interesting synchronization phenomena can be confirmed by computer simulations.
\end{abstract}

\section{Introduction}

Synchronization phenomena in coupled oscillatory systems are very good models to describe various higherdimensional nonlinear phenomena in the field of natural science. Studies on synchronization phenomena of coupled chaotic circuits are extensively carried out in various fields [1]-[10]. We consider that it is very important to investigate the phenomena related with chaos synchronization to realize future engineering application utilizing chaos. In our previous study, we have investigated the cross-coupled chaotic circuits and reported various interesting synchronization phenomena [11]-[13]. Additionally, in Figs. 1(a) and (b), we have investigated the three crosscoupled chaotic circuits and considered the small oscillations of three voltages are synchronized clearly in threephase. We can expect a large number of steady states with local three-phase of small oscillations. As we expected, we could find many different steady states[14].

We extend the previous study, in this study we consider five Shinriki-Mori chaotic circuits [15] cross-coupled via five inductors. The circuit is a special version of the ring considered in [12], however we have not investigated the phenomena in detail because we did not notice the phase differences of small oscillations until we noticed it in 2009 [13]. By computer simulations, we investigate the synchronization phenomena in detail, in particular, five-phase synchronizations of small oscillations are confirmed to be generated.

\section{Circuit Model}

Figure 2 shows the circuit model. In the circuit, five Shinriki-Mori chaotic circuits are cross-coupled via inductors $L_{2}$.

First, we approximate the $v-i$ characteristics of the nonlinear resistors consisting of the diodes by the following 3-segment piecewise-linear functions.

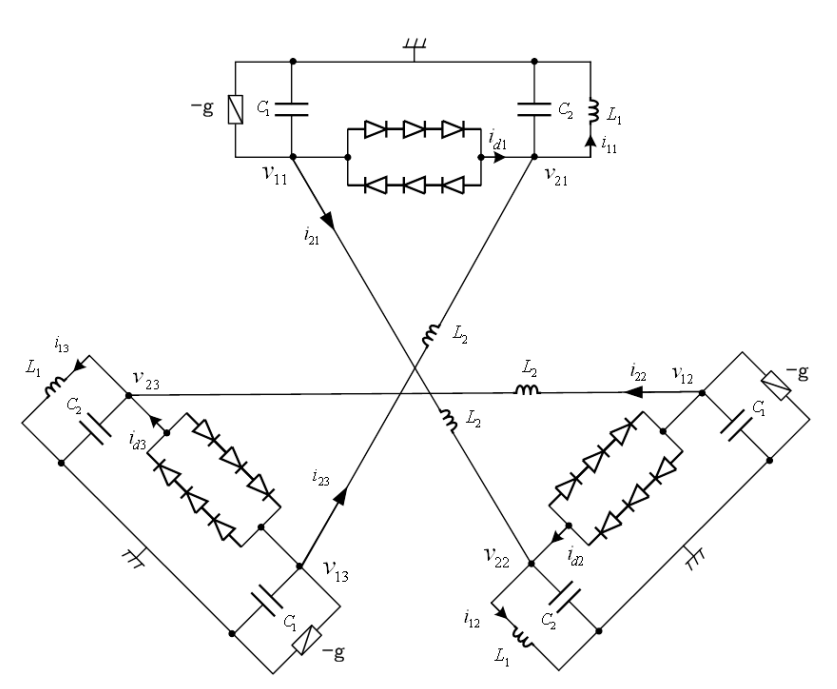

(a)
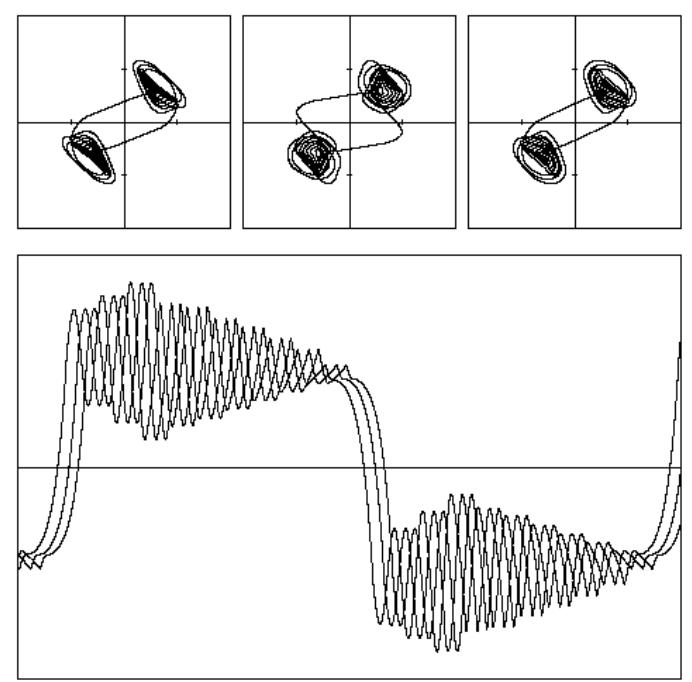

(b)

Figure 1: Three cross-coupled system.(a) Circuit model. (b)Upper figures show the attractor on $y_{1}-y_{2}$ phase plane, on $y_{1}-y_{3}$ phase plane and on $y_{2}-y_{3}$ phase plane from the left. Lower figure shows the time waveforms of $y_{1}, y_{2}$ and $y_{3}$. 


$$
i_{d n}= \begin{cases}G\left(v_{1 n}-v_{2 n}-V\right) & \left(v_{1 n}-v_{2 n}>V\right) \\ 0 & \left(\left|v_{1 n}-v_{2 n}\right| \leq V\right) \\ G\left(v_{1 n}-v_{2 n}+V\right) & \left(v_{1 n}-v_{2 n}<-V\right)\end{cases}
$$

$$
(n=1,2,3,4,5)
$$

The circuit equations are described as follows.

$$
\left\{\begin{aligned}
L_{1} \frac{d i_{1 n}}{d t} & =v_{2 n} \\
C_{1} \frac{d v_{1 n}}{d t} & =g v_{1 n}-i_{2 n}-i_{d n} \\
C_{2} \frac{d v_{2 n}}{d t} & =i_{d n}+i_{2, n-1}-i_{1 n} \\
L_{2} \frac{d i_{2 n}}{d t} & =v_{1 n}-v_{2, n+1} \\
(n & =1,2,3,4,5)
\end{aligned}\right.
$$

where $v_{20}=v_{25}$ and $v_{26}=v_{21}$. By using the following parameters

$$
\left\{\begin{array}{l}
\alpha=\frac{C_{2}}{C_{1}}, \beta=\sqrt{\frac{L_{1}}{C_{2}}} G, \gamma=\sqrt{\frac{L_{1}}{C_{2}}} g, \delta=\frac{L_{1}}{L_{2}}, \\
t=\sqrt{L_{1} C_{2}} \tau,
\end{array}\right.
$$

and variables

$$
\left\{\begin{array}{l}
i_{11}=\sqrt{\frac{C_{2}}{L_{1}}} V x_{1}, \quad i_{12}=\sqrt{\frac{C_{2}}{L_{1}}} V x_{2}, \quad i_{13}=\sqrt{\frac{C_{2}}{L_{1}}} V x_{3}, \\
v_{11}=V y_{1}, \quad v_{12}=V y_{2}, \quad v_{13}=V y_{3}, \\
v_{21}=V z_{1}, \quad v_{22}=V z_{2}, \quad v_{23}=V z_{3}, \\
i_{21}=\sqrt{\frac{C_{2}}{L_{1}}} V \omega_{1}, \quad i_{22}=\sqrt{\frac{C_{2}}{L_{1}}} V \omega_{2}, \quad i_{23}=\sqrt{\frac{C_{2}}{L_{1}}} V \omega_{3},
\end{array}\right.
$$

the normalized circuit equations are given as follows.

$$
\left\{\begin{aligned}
& \dot{x}_{n}= z_{n} \\
& \dot{y}_{n}= \alpha\left(\gamma y_{n}-\omega_{n}-\beta f\left(y_{n}-z_{n}\right)\right. \\
& \dot{z}_{n}= \beta f\left(y_{n}-z_{n}\right)+\omega_{n-1}-x_{n} \\
& \dot{\omega}_{n}= \delta\left(y_{n}-z_{n+1}\right) \\
&(n=1,2,3,4,5)
\end{aligned}\right.
$$

where $z_{20}=z_{25}$ and $z_{26}=z_{21}$. The nonlinear function $f(\cdot)$ corresponds to the $v-i$ characteristics of the nonlinear resistors consisting of the diodes and are assumed to be described by the following 3-segment piecewise-linear functions:

$$
\begin{gathered}
f\left(y_{n}-z_{n}\right)= \begin{cases}y_{n}-z_{n}-1 & \left(y_{n}-z_{n}>V\right) \\
0 & \left(\left|y_{n}-z_{n}\right| \leq V\right) \\
y_{n}-z_{n}+1 & \left(y_{n}-z_{n}<-V\right)\end{cases} \\
(n=1,2,3,4,5) .
\end{gathered}
$$

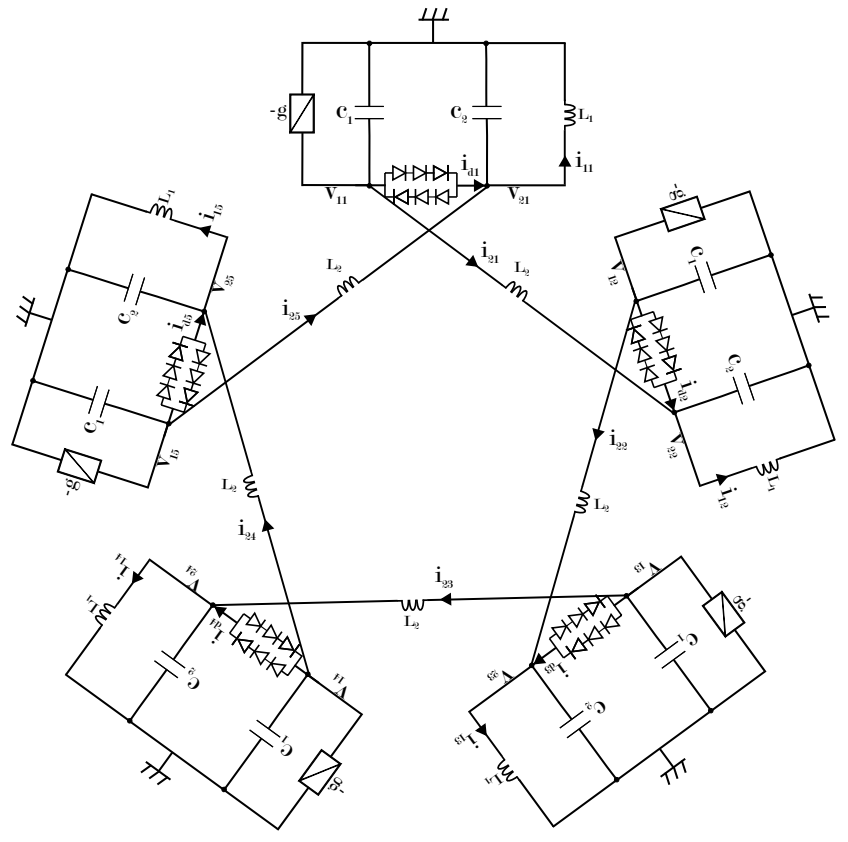

Figure 2: Circuit model.

\section{Equal Interval Phase Differences}

The proposed circuit model generates various synchronization states. Figure 3 shows some examples of attractors and magnify the time waveforms of the five voltages. These synchronization states are obtained for the same parameter set of $\alpha=2.0, \beta=4.0, \gamma=0.15$ and $\delta=0.004$ but with different initial conditions.

In Fig. 3(a), all five circuits seem to be synchronized in in-phase from the time waveforms, however, the attractors do not show in-phase synchronizations. The small oscillations of these five voltages are synchronized clearly in five-phase. This local five-phase synchronization is the reason of that the attractors in Fig. 3(a) do not show the in-phase synchronization completely in the phase space. Also we can understand why the shapes of the attractors show some phase differences in the first and the third quadrants of the phase space in Fig. 3(a). This result encouraged us to search many more coexisting steady states. As we expected, we could find many different steady states and some examples are shown in Figs. 3(b) and 3(c). The synchronization state depends on the initial conditions. In Figs. 3(b) and 3(c), the local five-phase state of the small oscillations varies by setting the difference initial conditions and the phase difference of these local five-phase of the small oscillations is shifted with the equal interval. Table 1 shows the phase differences of the voltage waveforms in Figs. 3.

We confirm combinations of the timing shift of the switching from minus to plus or from plus to minus with local five-phase of small oscillations make it possible to 
generate a large number of steady states.

Table 1: Phase difference of the voltages in Fig. 3.

\begin{tabular}{|c|c|c|c|}
\hline & (a) & (b) & (c) \\
\hline Phase difference & $3.57^{\circ}$ & $11.21^{\circ}$ & $27.57^{\circ}$ \\
\hline
\end{tabular}

\section{Unequal Interval Phase Differences}

Figures 4 shows some examples of attractors and magnify the time waveforms of the five voltages. These synchronization states are obtained for the same parameter set of $\alpha=2.0, \beta=4.0, \gamma=0.15$ and $\delta=0.004$ but with different initial conditions.

The amount of the timing shift of the switching from minus to plus or from plus to minus with local five-phase of small oscillations influences the shape of the attractors and synchronization states. In Fig. 3(a), the small oscillations of these five voltages are synchronized clearly in five-phase and in Figs. 3(b), (c), local five-phase of small oscillations rise up at equal interval. By contrast, in Fig. 4, each local small oscillations rise up at unequal interval.

These result we can expect a large number of steady states with local five-phase of small oscillations.

\section{Conclutions}

In this study, we have investigated the synchronization phenomena observed from five simple chaotic circuits cross-coupled by inductors. The detailed investigation of time waveforms clarified several interesting phenomena.

Investigating the coexistence of the states and statistical analysis of the observed phenomena are our important future work as well as more detailed explanation of the mechanism of the generations.

\section{Acknowledgment}

This work was partly supported by JSPS Grant-in-Aid for Young Scientists 23700269.

\section{References}

[1] Ch. von der Malsbuurg and J. Buhmann, "Sensory Segmentation with Coupled Neural Oscillators," Biol. Cybern., vol. 67, pp. 233-242, 1992.

[2] N. Platt, E.A. Spiegel and C. Tresser, "On-Off Intermittency: A Mechanism for Bursting," Phys. Rev. Lett., vol. 70, no. 3, pp. 279-282, 1993.

[3] P. Ashwin, J. Buescu and I. Stewart, "Bubbling of Attractors and Synchronization of Chaotic Oscillators," Phys. Lett. A,193, pp. 126-139, 1994.

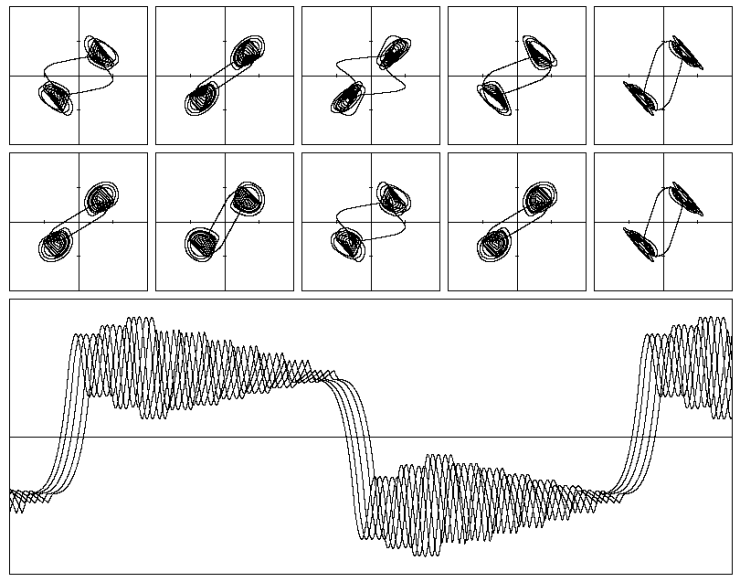

(a)

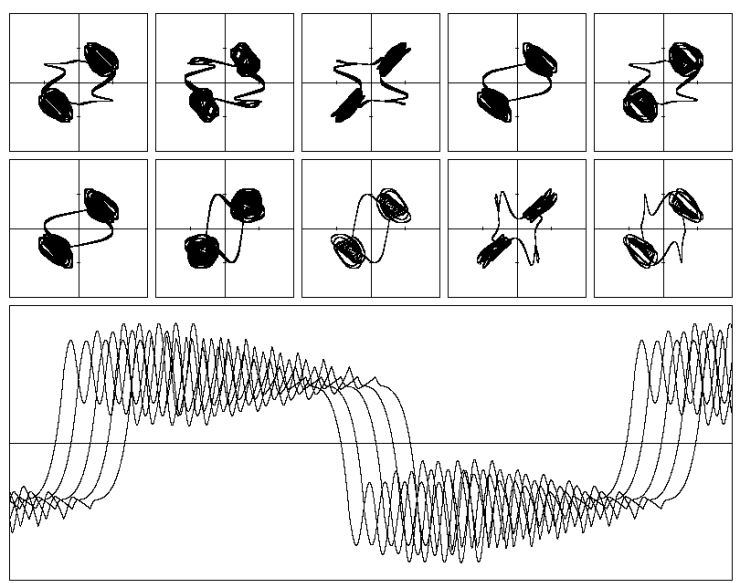

(b)

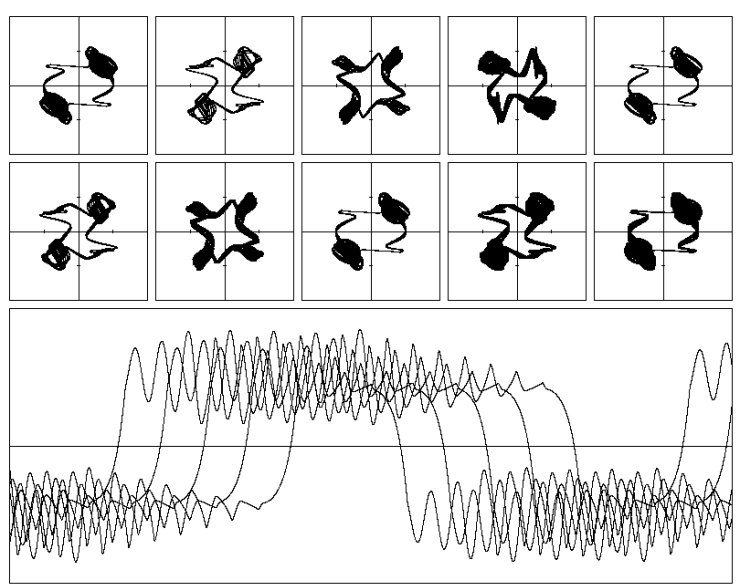

(c)

Figure 3: State transition phenomena around for constant phase difference. Upper figures show the attractor on $y_{1}-y_{2}, y_{1}-y_{3}$, $y_{1}-y_{4}, y_{1}-y_{5}$ and $y_{2}-y_{3}$ phase plane from the left. Middle figures show the attractor on $y_{2}-y_{4}, y_{2}-y_{5}, y_{3}-y_{4}, y_{3}-y_{5}$ and $y_{4}-y_{5}$ phase plane from the left. Lower figure shows the magnified time waveforms of $y_{1}, y_{2}, y_{3}, y_{4}$ and $y_{5}$. 


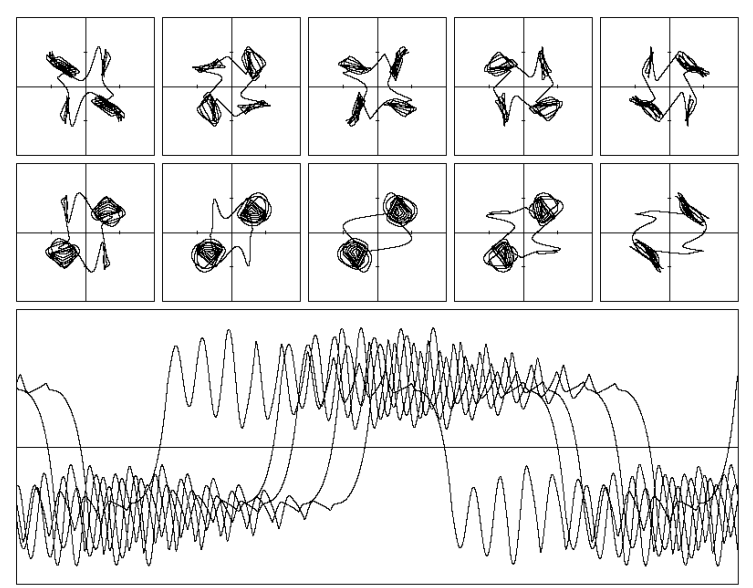

(a)

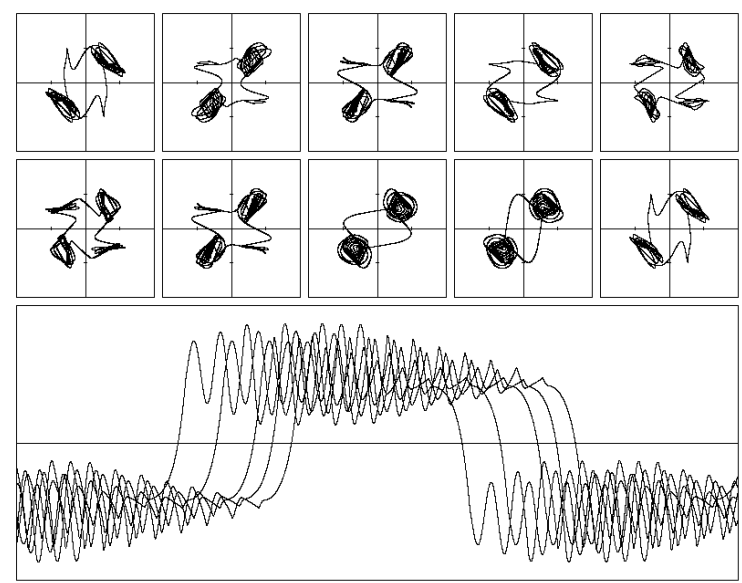

(b)

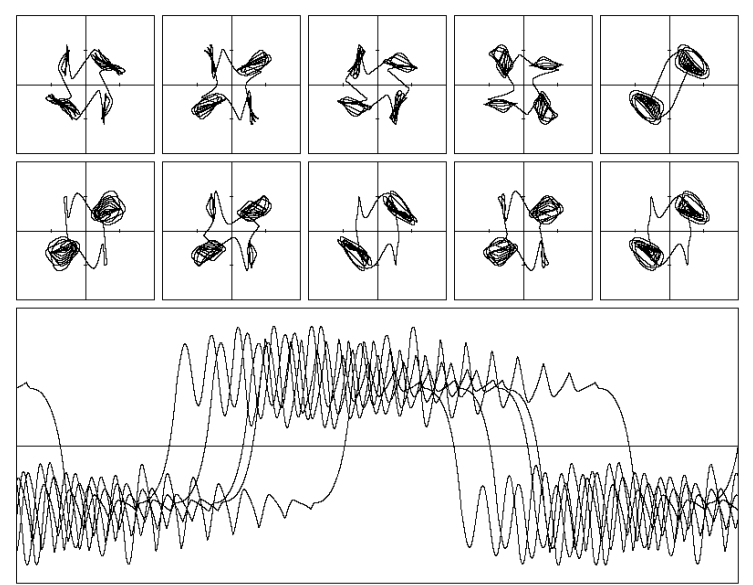

(c)

Figure 4: State transition phenomena around for indeterminate phase difference. Upper figures show the attractor on $y_{1}-y_{2}$, $y_{1}-y_{3}, y_{1}-y_{4}, y_{1}-y_{5}$ and $y_{2}-y_{3}$ phase plane from the left. Middle figures show the attractor on $y_{2}-y_{4}, y_{2}-y_{5}, y_{3}-y_{4}$, $y_{3}-y_{5}$ and $y_{4}-y_{5}$ phase plane from the left. Lower figure shows the magnified time waveforms of $y_{1}, y_{2}, y_{3}, y_{4}$ and $y_{5}$.
[4] E. Ott and J.C. Sommerer, "Blowout Bifurcations: the Occurrence of Riddled Basins and On-Off Intermittency," Phys. Lett. A,199, pp. 39-47, 1994.

[5] Y. Nishio and A. Ushida, "Spatio-Temporal Chaos in Simple Coupled Chaotic Circuits," IEEE Trans. Circuits Syst. I, vol. 42, no. 10, pp. 678-686, 1995.

[6] N.F. Rulkov and M.M. Sushchik, "Robustness of Synchronized Chaotic Oscillations," Int. J. Bifurcation and Chaos, vol. 7, no. 3, pp. 625-643, 1997.

[7] M. Wada, Y. Nishio and A. Ushida, "Analysis of Bifurcation Phenomena in Two Chaotic Circuits Coupled by an Inductor," IEICE Trans. Fundamentals, vol. E80-A, no. 5, pp. 869-875, 1997.

[8] Y. Nishio and A. Ushida, "Chaotic Wandering and its Analysis in Simple Coupled Chaotic Circuits," IEICE Trans. Fundamentals, vol. E85-A, no. 1, pp. 248-255, 2002.

[9] G. Abramson,V.M. Kenkre and A.R. Bishop, "Analytic Solutions for Nonlinear Waves in Coupled Reacting Systems," Physica A: Statistical Mecanics and its Applications, vol. 305, no. 3-4, pp. 427-436, 2002.

[10] I. Belykh, M. Hasler, M. Lauret and H. Nijmeijer, "Synchronization and Graph Topology," Int. J. Bifurcation and Chaos, vol. 15, no. 11, pp. 3423-3433, 2005.

[11] Y. Uchitani and Y. Nishio, "Investigation of State Transition Phenomena in Cross-Coupled Chaotic Circuits,' Proc. of ISCAS'08, pp. 2394-2397, May 2008.

[12] Y. Uchitani and Y. Nishio, "Synchronization Patterns Generated in a Ring of Cross-Coupled Chaotic Circuits," Proc of IJCNN'08, pp. 3854-3859, Jun. 2008.

[13] Y. Uchitani and Y. Nishio, "Synchronization of Small Oscillations in Cross-Coupled Chaotic Circuits," Proc of ISCAS'09, pp. 2629-2632, May 2009.

[14] N. Kageyama, Y. Uwate and Y. Nishio, "Detailed Investigation of Synchronizations in Three CrossCoupled Chaotic Circuits," Proc. of NCSP'13, pp. 628-631, Mar. 2013.

[15] M. Shinriki, M. Yamamoto and S. Mori, "Multi-Mode Oscillations in a Modified van der Pol Oscillator Containing a Positive Nonlinear Conductance," Proc. of IEEE, vol. 69, pp. 394-395, 1981. 